\title{
BIOELECTRICAL IMPEDANCE COMPONENTS AND THE MASS AND STRENGTH OF UPPER LIMB SKELETAL MUSCLES IN YOUNG ADULTS
}

original paper

(c) University School of Physical Education in Wroclaw

DOI: https://doi.org/10.5114/hm.2020.95989

\section{MAŁGORZATA KOŁODZIEJ, DAWID KOŹLENIA, KATARZYNA KOCHAN-JACHEĆ, JAROSEAW DOMARADZKI}

Faculty of Physical Education, University School of Physical Education in Wrocław, Wrocław, Poland

\begin{abstract}
Purpose. The aim of this study was to evaluate bioelectrical impedance components as markers of mass and strength of upper limb skeletal muscles in young adults.

Methods. The study involved 89 healthy adults aged 19-26 years. Body height and mass and hand grip strength were measured. Resistance, reactance, and phase angle were established with the bioelectrical impedance analysis. A prediction equation was used to estimate appendicular skeletal muscle mass. Relationships of the impedance components with the proportion of skeletal muscles in the total appendicular muscle mass and with the strength and functional quality indices were examined. Results. Differences between women and men for all the impedance components and relationships between these components and appendicular skeletal muscle mass and strength have been shown. Higher muscle mass and hand grip strength values were registered in males than in females, but the percentage of skeletal muscles in the appendicular muscle mass and the muscle strength index were found to be lower in men. No differences were found in the quality of the upper extremity skeletal muscles between the gender groups.
\end{abstract}

Conclusions. Impedance components are good identifiers of differences in the mass and strength of the appendicular skeletal muscles in healthy adults. Phase angle and reactance are strong predictors of skeletal muscle participation in total limb muscle mass. Higher strength generated by unit muscle mass is identified by higher reactance, which assesses the quality of soft tissues.

Key words: bioelectrical impedance analysis, appendicular skeletal muscle, hand grip strength, muscle quality index, young adults

\section{Introduction}

Over 600 individual skeletal muscles take part in the essential mechanical, structural, and metabolic functions of the entire body. Appendicular skeletal muscles constitute the largest proportion of the overall skeletal muscle mass [1]. The mass of the skeletal muscles increases with age until the third decade of life and subsequently their gradual atrophy is observed, which accelerates with age $[2,3]$. The decline in muscle mass reduces muscle strength and general physical fitness [4]. Even though muscle mass explains most of the variances in muscle strength, it has been found that the decline in this strength is greater and quicker than that in muscle mass and that interventions increasing muscle mass do not necessarily raise muscle strength. This phenomenon may be associated with changes in muscle composition and quality [3, 5].

The practical application of the reference methods of estimating the skeletal muscle mass, such as computerized tomography, magnetic resonance imaging, and dual-energy X-ray absorptiometry (DXA), is limited because of the high cost, the need for trained operating personnel, and the long-term radiation exposure [2, 6]. A cheaper and safer method, more convenient to operate and apply, is bioelectrical impedance analysis (BIA) [1]. The drawback of this method is that it does not directly measure body composition. The water content and

Correspondence address: Małgorzata Kołodziej, Faculty of Physical Education, University School of Physical Education in Wrocław, al. I.J. Paderewskiego 35, 51-612 Wrocław, Poland, e-mail: malgorzata.kolodziej@awf.wroc.pl

Received: February 6, 2020

Accepted for publication: May 20, 2020

Citation: Kołodziej M, Koźlenia D, Kochan-Jacheć K, Domaradzki J, Bioelectrical impedance components and the mass and strength of upper limb skeletal muscles in young adults. Hum Mov. 2020;21(4):111-117; doi: https://doi.org/10.5114/ hm.2020.95989 
fat-free and fat mass are estimated by using equations that, generated on the basis of results of tests in a particular population, may incorrectly estimate the body composition of another population [7-9]. The disputability of the accuracy of the BIA prediction equations can be limited through the use of directly measured electrical impedance components in the analysis of body composition changes. Chemical processes in muscle tissues can change their electrical properties, therefore measuring electrical impedance components seems to be a useful way to assess muscle quality.

The aim of our study was to evaluate raw bioelectrical impedance data obtained from BIA measurements, as markers of the mass and strength of upper limb skeletal muscles in young adults.

\section{Material and methods}

\section{Participants}

Overall, 89 persons (including 41 men and 48 women) aged $19-26$ years $(20.7 \pm 1.0$ years) who volunteered for the free tests owing to university announcements were studied. The participants were informed about the purpose and methods of this study and about the experiment risk. All the subjects declared regularly undertaken physical activity, but without competitive training (no sports class), and no injury in the period of 6 weeks prior to the study. In accordance with the BIA analyser manufacturer's recommendations, the following exclusion criteria were used: the presence of a metal or electronic implant, limb amputation, and pregnancy.

\section{Measures and procedures}

Body height and mass were measured by means of electronic scales with an integrated digital stadiometer SECA 764 (Seca GmbH \& Co. KG, Germany). The measurement accuracy was $0.1 \mathrm{~kg}$ for mass and $0.1 \mathrm{~cm}$ for height. Impedance was evaluated by using a multifrequency analyser TANITA MC 180 MA (Tanita Corporation, Japan) at a $50-\mathrm{kHz}$ current frequency. The characteristics of the device and the applied BIA measurement procedures were described in a previous report [10]. All subjects were asked to refrain from eating, drinking, and physical activity for at least 3 hours before the BIA assessment. The resistance (R) measurements were normalized to body height $(\mathrm{Ht})$ to obtain the resistance index $\left(\mathrm{Ht}^{2} / \mathrm{R}\right)$. The sum of the appendicular muscle masses (AMM) of the particular limbs, estimated by using the manufacturer's proprietary equation, was assumed as the total AMM. The appendicular skeletal muscle mass (ASMM) was estimated with the Kyle's equation developed for healthy Caucasians aged 22-95 years [11]:

$$
\begin{gathered}
\mathrm{ASMM}=-4.211+\left(0.267^{*} \mathrm{Ht}^{2} / \mathrm{R}\right)+\left(0.095^{*} \mathrm{Wt}\right)+ \\
\left(0.058^{*} \mathrm{X}_{\mathrm{c}}\right)+\left(1.909^{*} \mathrm{sex}\right)+\left(-0.012^{*} \text { age }\right)
\end{gathered}
$$

where: ASMM - appendicular skeletal muscle mass $(\mathrm{kg}), \mathrm{Ht}$ - height $(\mathrm{cm}), \mathrm{R}$ - resistance $(\Omega), \mathrm{Ht}^{2} / \mathrm{R}$ - resistance index $\left(\mathrm{cm}^{2} / \Omega\right)$, Wt - weight $(\mathrm{kg}), \mathrm{X}_{\mathrm{c}}$ - reactance $(\Omega)$, sex: men $=1$ and women $=0$.

In order to minimize the differences stemming from the inter-subject variability, the AMM value and the ASMM value were scaled to body height [12]:

$$
\begin{aligned}
\operatorname{AMMI}\left(\mathrm{kg} / \mathrm{m}^{2}\right) & =\operatorname{AMM}(\mathrm{kg}) / \mathrm{Ht}^{2}\left(\mathrm{~m}^{2}\right) \\
\operatorname{ASMMI}\left(\mathrm{kg} / \mathrm{m}^{2}\right) & =\operatorname{ASMM}(\mathrm{kg}) / \mathrm{Ht}^{2}\left(\mathrm{~m}^{2}\right)
\end{aligned}
$$

where: AMMI - appendicular muscle mass index, ASMMI - appendicular skeletal muscle mass index, Ht - height.

Hand grip strength (HGS) was measured with an accuracy of $1 \mathrm{~kg}$ by means of a JAMAR (Sammons Preston Rolyan, USA) hydraulic hand dynamometer with an adjustable handle set to position 2 . The recommendations of the American Society of Hand Therapists, i.e. the subject seated, the shoulder adducted and neutrally rotated, the elbow flexed at $90^{\circ}$, and the forearm and the wrist in neutral position, were adopted. The participants were asked to perform 2 maximum grip strength tests for alternately the left hand and the right hand. Each of the tests lasted 3 seconds and the inter-measurement interval was 15-20 seconds. The average of the best results for the two limbs was adopted as the HGS value.

The ratio of HGS to the estimated AMM was assumed as the muscle strength index while the ratio of HGS to the estimated ASMM constituted the appendicular skeletal muscle quality index [12].

\section{Statistical analysis}

All statistical analyses were performed with the Statistca 13.1 software [13]. All results are expressed as means \pm standard deviations, and 95\% confidence intervals were calculated. The Shapiro-Wilk test was used to verify the normality of variable distribution. The correlations between the variables were tested with Pearson's correlation coefficient $(r)$. The differences 
between the gender groups were evaluated by applying the Student's $t$-test to the mean difference for the independent samples. The prediction of the impedance parameters for the proportion of the skeletal muscles in the total AMM was evaluated by using stepwise multiple regression (entry probability of $\mathrm{F}$ was 0.05 and removal was 0.10). The statistical significance of the results was set at $p<0.05$.

\section{Ethical approval}

The research related to human use has complied with all the relevant national regulations and institutional policies, has followed the tenets of the Declaration of Helsinki, and has been approved by the Research Ethics Committee of the institution of the authors.

\section{Informed consent}

Informed consent has been obtained from all individuals included in this study.

\section{Results}

The descriptive statistics of the subjects and the differences between the men and the women are presented in Table 1. The body height and mass, the phase angle, the resistance index, and the estimated AMM and ASMM were significantly higher in males, whereas the total impedance, resistance, and reactance values $(p<0.01)$ were higher in females (Table 1$)$.

The resistance index among men was 35\% higher than $\mathrm{Ht}^{2} / \mathrm{R}$ in women, which corresponds to the relative ASMM greater by about $1.9 \mathrm{~kg} / \mathrm{m}^{2}$ than in women. Even though men were characterized by greater AMM and ASMM, the proportion of the skeletal muscles in AMM was larger by nearly $6 \%$ in women.

The correlation between the impedance parameters and the ASMM proportion in the overall AMM was tested with stepwise multiple regression analysis. The resistance index was not included in the analysis since both AMM and ASMM had been estimated on its basis (it had been the dominant predictor of the two variables). The mass, height, resistance (strongly linearly correlated with one another), and the unvaried age of the subjects were not taken into account in the regression model. The best predictors of the ASMM proportion in AMM were sex $($ men $=1$, women $=0)$, reactance, and phase angle. The model explained 75\% of the variation of the ASMM in the AMM, and the standard estimation error amounted to 0.018 (Table 2). No mutual correlations between the model coefficients $(r<0.0001)$ were observed and the residuals were found to be normally distributed. Sex was the strongest predictor of the ASMM level, but the positive effects of reactance and phase angle were also significant.

Table 1. Descriptive characteristics of the study participants, mean $\pm S D$ (95\% CI)

\begin{tabular}{|c|c|c|c|c|}
\hline Characteristics & Total $(n=89)$ & Men $(n=41)$ & Women $(n=48)$ & $p$ \\
\hline Age (years) & $20.7 \pm 1.0(20.4-20.9)$ & $21.0 \pm 1.2(20.6-21.4)$ & $20.4 \pm 0.6(20.2-20.6)$ & 0.004 \\
\hline Height (cm) & $174.4 \pm 9.5(172.4-176.4)$ & $182.2 \pm 5.7(180.4-184.0)$ & $167.8 \pm 6.4(165.9-169.6)$ & $<0.001$ \\
\hline Weight (kg) & $68.4 \pm 13.1(65.67-71.1)$ & $79.4 \pm 8.2(76.8-82.0)$ & $59.0 \pm 7.8(56.7-61.2)$ & $<0.001$ \\
\hline $\operatorname{BMI}\left(\mathrm{kg} / \mathrm{m}^{2}\right)$ & $22.3 \pm 2.5(21.8-22.8)$ & $23.9 \pm 2.0(23.3-24.5)$ & $20.91 \pm 2.1(20.3-21.5)$ & $<0.001$ \\
\hline $\mathrm{Z}(\Omega)$ & $626.2 \pm 105.7(603.9-648.5)$ & $536.2 \pm 49.4(520.6-551.8)$ & $703.0 \pm 75.8(681.0-725.0)$ & $<0.001$ \\
\hline $\mathrm{R}(\Omega)$ & $622.3 \pm 105.6(600.0-644.5)$ & $532.3 \pm 49.2(516.8-547.8)$ & $699.2 \pm 75.6(677.2-721.1)$ & $<0.001$ \\
\hline $\mathrm{X}_{\mathrm{c}}(\Omega)$ & $69.5 \pm 8.6(67.7-71.3)$ & $64.8 \pm 6.3(62.8-66.8)$ & $73.6 \pm 8.2(71.2-75.9)$ & $<0.001$ \\
\hline $\operatorname{PhA}\left({ }^{\circ}\right)$ & $6.45 \pm 0.69(6.31-6.60)$ & $6.95 \pm 0.46(6.81-7.10)$ & $6.03 \pm 0.57(5.86-6.19)$ & $<0.001$ \\
\hline $\mathrm{Ht}^{2} / \mathrm{R}\left(\mathrm{cm}^{2} / \Omega\right)$ & $51.0 \pm 12.6(48.3-53.6)$ & $62.9 \pm 6.8(60.8-65.1)$ & $40.7 \pm 5.0(39.3-42.2)$ & $<0.001$ \\
\hline AMM (kg) & $23.9 \pm 6.4(22.5-25.2)$ & $30.4 \pm 2.5(29.6-31.2)$ & $18.3 \pm 1.7(17.8-18.8)$ & $<0.001$ \\
\hline $\operatorname{AMMI}\left(\mathrm{kg} / \mathrm{m}^{2}\right)$ & $7.72 \pm 1.45(7.42-8.03)$ & $9.15 \pm 0.65(8.95-9.36)$ & $6.50 \pm 0.53(6.35-6.66)$ & $<0.001$ \\
\hline ASMM (kg) & $20.6 \pm 5.1(19.5-21.6)$ & $25.6 \pm 2.3(24.8-26.3)$ & $16.3 \pm 1.8(15.8-16.8)$ & $<0.001$ \\
\hline $\operatorname{ASMMI}\left(\mathrm{kg} / \mathrm{m}^{2}\right)$ & $6.66 \pm 1.09(6.43-6.89)$ & $7.69 \pm 0.57(7.51-7.87)$ & $5.78 \pm 0.49(5.64-5.92)$ & $<0.001$ \\
\hline ASMM/AMM & $0.87 \pm 0.04(0.87-0.86)$ & $0.84 \pm 0.02(0.84-0.85)$ & $0.89 \pm 0.03(0.88-0.90)$ & $<0.001$ \\
\hline HGS (kg) & $44.1 \pm 11.6(41.7-46.6)$ & $54.8 \pm 7.0(52.6-57.0)$ & $35.0 \pm 5.0(33.5-36.4)$ & $<0.001$ \\
\hline HGS/AMM & $1.86 \pm 0.22(1.82-1.91)$ & $1.81 \pm 0.20(1.74-1.87)$ & $1.91 \pm 0.22(1.85-1.98)$ & 0.022 \\
\hline HGS/ASMM & $2.15 \pm 0.24(2.10-2.20)$ & $2.15 \pm 0.24(2.08-2.23)$ & $2.15 \pm 0.24(2.08-2.22)$ & 0.999 \\
\hline
\end{tabular}

$S D$ - standard deviation, CI - confidence interval, BMI - body mass index, $\mathrm{Z}$ - impedance, $\mathrm{R}$ - resistance, $\mathrm{X}_{\mathrm{c}}-$ reactance, $\mathrm{PhA}$ - phase angle, $\mathrm{Ht}$ - height, $\mathrm{Ht}^{2} / \mathrm{R}$ - resistance index, AMM - appendicular muscle mass, AMMI - appendicular muscle mass index, ASMM - appendicular skeletal muscle mass, ASMMI - appendicular skeletal muscle mass index, HGS - hand grip strength, HGS/AMM - muscle strength index, HGS/ASMM - muscle quality index 
Table 2. Multiple linear regression model for ASMM/AMM

\begin{tabular}{ccccccc} 
& Predictor & $\beta(S E)$ & $b(S E)$ & $p$ & $\mathrm{R}^{2}$ cumulative & \\
\hline & Intercept & & $0.671(0.024)$ & $<0.001$ & & $\mathrm{R}^{2}=0.750$ \\
ASMM/AMM & $\mathrm{Sex}$ & $-0.977(0.094)$ & $-0.068(0.007)$ & $<0.001$ & 0.670 & $\mathrm{~F}_{(3,85)}=84.921, p<0.001$ \\
& $\mathrm{PhA}$ & $0.544(0.081)$ & $0.027(0.004)$ & $<0.001$ & 0.554 & $S E E=0.018$ \\
& $\mathrm{X}_{\mathrm{c}}$ & $0.183(0.071)$ & $0.001(0.0003)$ & 0.011 & 0.410 & $S$ \\
\hline
\end{tabular}

ASMM - appendicular skeletal muscle mass, AMM - appendicular muscle mass, sex: men = 1 and women $=0$, PhA - phase angle, $\mathrm{X}_{\mathrm{c}}$ - reactance, $\beta$ - standardized regression coefficient, $S E$ - standard error, $b$ - unstandardized regression coefficient, $\mathrm{R}^{2}$ - coefficient of determination; $\mathrm{F}_{(3,85)}, p$ - Fisher test, $S E E$ - standard error of estimation

The strength of the muscles of the upper limbs was evaluated by the ratio of the mean grip strength of both hands to the estimated AMM (HGS/AMM) while the functional quality of the upper limb skeletal muscles was assessed with the ratio of the grip strength to the skeletal muscle mass (HGS/ASMM).

As expected, the absolute HGS in men was significantly greater than in women, but the HGS relative to AMM, i.e. the HGS/AMM index of the upper limb muscle strength, was significantly higher among females. The value of the HGS/ASMM index of the quality of the upper limb skeletal muscles was the same in both gender groups (Table 1). Correlations between reactance and HGS/AMM $(r=0.345, p=0.001)$ and between reactance and HGS/ASMM $(r=0.265, p=$ 0.012 ) were observed, but no correlation of the two indices with the other bioelectrical parameters (R, phase angle) was found.

\section{Discussion}

Using the impedance components measured by means of the BIA method, we focused on the upper limb muscles, considering that the forearm, which constitutes $1.3 \%$ of the body mass, amounts to $25.0 \%$ the whole body impedance [14]. Moreover, the appendicular muscles, whose proportion in the total skeletal muscle mass is the largest, are a major determinant of motion generation and physical fitness $[15,16]$.

The strongest independent predictor in models predicting muscle mass, including ASMM, is the resistance index $\left(\mathrm{Ht}^{2} / \mathrm{R}\right)$, which constitutes a measure of the body electrical conductance $[9,11]$. In our study, the resistance index of the young men was 1.6 times higher than the $\mathrm{Ht}^{2} / \mathrm{R}$ of the young women. The higher electrical conductance characteristic of men is due to the greater mass of electrically conducting muscle tissues in comparison with women [7]. The ASMM of women amounted to over $60 \%$ of the ASMM of men, which confirmed the DXA test results obtained by Kyle et al. [11] and the outcomes of our earlier research performed among elderly persons [10]. The differences in ASMM and AMM between women and men were also preserved in the case of values corrected for the square of body height (AMMI and ASMMI), which corroborates the findings that in similar age groups, men have higher absolute and relative fat-free mass values, including muscle mass, than women $[9,17]$. Using the ASMM/ AMM ratio, we established the proportion of skeletal muscles in AMM in the group of the investigated young individuals to be significantly smaller in men than in women, which was not expected considering the earlier findings indicating the much greater muscle mass corrected for the square of body height in men. We have not found reports which would corroborate our findings, but there are some studies suggesting that the proportion of skeletal muscles in the muscle mass is not greater in men than in women. Carnevale et al. [18], in a study covering older people, observed that men and women did not differ in their relative upper extremity muscle mass.

On the basis of the stepwise regression results, we found sex to be the strongest indicator of the ASMM level (pointing at a higher ASMM/AMM ratio for women than for men); however, the positive effects of reactance and phase angle were also significant. The lower percentage of skeletal muscles in the overall AMM in men can be ascribed to the lower reactance registered for men in comparison with that reported for women. The difference in the reactance value between men and women was even more significant after the measured reactance values were corrected for body height or body mass index. Reactance is connected with the capillary capacity of cell membranes [7, 19]. The lower reactance that we observed in men in comparison with women could be linked to a lower cellular mass due to lower intracellular hydration or to lower cell (including skeletal muscle cells) membrane functionality $[19,20]$.

Also, phase angle is regarded as a measure of the quality of soft tissues. Its value depends on the size and 
number of cells with integral cell membranes and on the distribution of intra- and extracellular water [20, 21]. It has been confirmed that phase angle values correlate, among others, with age, sex, state of nutrition, and physical activity [7, 22]. The phase angle increases up to early adulthood and then gradually decreases in later years [23, 24]. In healthy individuals, the phase angle usually ranges between $5^{\circ}$ and $7^{\circ}[21,22]$. In the studied group, we did not register any cases of a too low phase angle value. The minimum values amounted to $6.0^{\circ}$ for men and to $5.1^{\circ}$ for women. The significantly higher phase angle values in males are mainly due to the larger number of cells, associated with the greater muscle mass of men [22]. After phase angle was corrected for body mass or body mass index, the differences in phase angle between men and women became not significant.

The relationships between the bioelectrical impedance components and the electrical properties of tissues and cells are indicators not only of body composition, including skeletal muscle mass, but also of the quality of the latter, which translates into muscle strength and functionality $[1,21]$. It is suggested that the quality of muscles is a more important predictor of muscle strength than the volume of muscles in young and older men and women [25].

As expected, the obtained results showed a difference in muscle strength (evaluated with HGS) between men and women, which had been repeatedly reported for the groups of muscles of the whole body and the appendicular muscles in young people and adults at different ages [26, 27]. It has been found that the differences stem from the strong influence of body size on muscle strength. Hence, assessments of muscular functions, most often based on limb strength measurements, are distorted by the influence of the total muscle mass, which implies that muscle strength indices independent of body size should be used [28]. We assumed HGS normalized to AMM as the muscle strength evaluating parameter. Using this index (HGS/AMM), we found the muscle strength in men to be lower than in women, despite the fact that, as mentioned earlier, males showed much stronger hand grip than females. The stated earlier larger percentage of skeletal muscles in AMM probably generated greater unit muscular strength in women. The recorded significantly higher reactance in women than in men, as well as the positive correlation of the reactance with the HGS/AMM values suggest that the better muscular functionality observed in females can also be connected with the probably better (than in males) nutrition status of the cells of the upper limb muscles.
Even though reactance positively correlated with the HGS/ASMM ratio, the functional quality of the upper limb skeletal muscles, assessed with the HGS/ ASMM ratio, was the same in both gender groups. Also Alizadehkhaiyat et al. [29], when studying people aged 19-49 years, did not find significant differences between men and women in the quality of the arms muscles, evaluated with the strength measured in 5 different planes and normalized to the skeletal muscle mass, although they determined significant differences in the absolute strength of the arms between the sexes. In another study covering adults, but in a wider range of age (18-78 years), Charlier et al. [12] also did not observe any differences between women and men in HGS corrected for the total skeletal muscle mass.

It has been found that the mass and strength of muscles gradually decrease after the the age of 20 [17] and the process accelerates with age $[2,3,30]$. Fraser et al. [27] revealed that the muscle quality and strength assessed in childhood and early youth could be a good predictor of the condition of the muscles in later periods of the ontogenesis. Hence, young adulthood seems to be the key period for monitoring and intervention in order to reduce the risk of asthenia and chronic diseases, especially cardiometabolic ones. Health behaviours aimed at the care of muscle quality and strength are the key strategy for healthy ageing.

The main limitation of our study is that it is a surveillance study, which does not prove a causal relationship, and its results can be generalized only to a population of healthy young adults as it covered sports university students (aged 19-26 years), physically active (but not sports-wise classified). Secondly, no evaluation was carried out of the parameters which could have a bearing on the relationship between muscle mass and muscle function, such as protein intake or physical activity. Despite the limitations, we believe that this project extends the existing knowledge of the possibilities to identify muscle mass and quality and can form an important basis for orienting further research towards evaluating BIA potential for monitoring changes in body composition without the use of predictive equations.

\section{Conclusions}

The results of our study indicate that the impedance components measured directly during body composition estimation through BIA can be used to assess not only muscle mass, but also the strength and quality of the limb skeletal muscles. Phase angle and reactance have been shown to constitute strong predictors of the 
skeletal muscle participation in the total limb muscle mass. And, moreover, the higher strength generated by unit muscle mass is identified by higher reactance, which assesses the quality of soft tissues. The results suggest that muscle strength is not completely explained by muscle mass itself, but also by skeletal muscle mass proportion and quality.

The use of the impedance components for the preventive monitoring of changes in muscle mass, strength, and quality seems to be justified considering the easy, quick, and inexpensive method of measuring them provided by BIA.

The results of our study led to the formulation of several hypotheses concerning the causes of the smaller proportion of skeletal muscles in the muscle mass of the upper limbs and the lower muscular strength per unit AMM in men than in women. The hypotheses provide a sound basis for expanding the research on the quality of skeletal muscles.

\section{Acknowledgements}

The authors thank all volunteers for their collaboration during the study. This research did not receive any specific grant from funding agencies in the public, commercial, or not-for-profit sectors.

\section{Disclosure statement}

No author has any financial interest or received any financial benefit from this research.

\section{Conflict of interest}

The authors state no conflict of interest.

\section{References}

1. Heymsfield SB, Gonzalez MC, Lu J, Jia G, Zheng J. Skeletal muscle mass and quality: evolution of modern measurement concepts in the context of sarcopenia. Proc Nutr Soc. 2015;74(4):355-366; doi: 10.1017/ S0029665115000129.

2. Janssen I, Heymsfield SB, Wang ZM, Ross R. Skeletal muscle mass and distribution in 468 men and women aged 18-88 yr. J Appl Physiol. 2000;89(1):81-88; doi: 10.1152/jappl.2000.89.1.81.

3. Goodpaster BH, Carlson CL, Visser M, Kelly DE, Scherzinger A, Harris TB, et al. Attenuation of skeletal muscle and strength in the elderly: the Health ABC Study. J Appl Physiol. 2001;90(6):2157-2165; doi: 10.1152/ jappl.2001.90.6.2157.

4. Fried LP, Tangen CM, Walston J, Newman AB, Hirsch C, Gottdiener J, et al. Frailty in older adults: evidence for a phenotype. J Gerontol A Biol Sci Med Sci. 2001;56(3): M146-M156; doi: 10.1093/gerona/56.3.M146.

5. Moore AZ, Caturegli G, Metter EJ, Makrogiannis S, Resnick SM, Harris TB, et al. Difference in muscle quality over the adult life span and biological correlates in the Baltimore Longitudinal Study of Aging. J Am Geriatr Soc. 2014;62(2):230-236; doi: 10.1111/jgs.12653.

6. Buckinx F, Landi F, Cesari M, Fielding RA, Visser M, Engelke K, et al. Pitfalls in the measurement of muscle mass: a need for a reference standard. J Cachexia Sarcopenia Muscle. 2018;9(2):269-278; doi: 10.1002/ jcsm.12268.

7. Kyle UG, Bosaeus I, De Lorenzo AD, Deurenberg P, Elia M, Gómez JM, et al. Bioelectrical impedance analysis - Part I: Review of principles and methods. Clin Nutr. 2004;23(5):1226-1243; doi: 10.1016/j.clnu.2004.06.004.

8. Bosy-Westphal A, Jensen B, Braun W, Pourhassan M, Gallagher D, Müller MJ. Quantification of whole-body and segmental skeletal muscle mass using phase-sensitive 8-electrode medical bioelectrical impedance devices. Eur J Clin Nutr. 2017;71(9):1061-1067; doi: 10.1038/ejcn.2017.27.

9. Janssen I, Heymsfield SB, Baumgartner RN, Ross R. Estimation of skeletal muscle mass by bioelectrical impedance analysis. J Appl Physiol. 2000;89(2):465-471; doi: 10.1152/jappl.2000.89.2.465.

10. Kołodziej M, Ignasiak Z. Changes in the bioelectrical impedance parameters estimating appendicular skeletal muscle mass in healthy older persons. Aging Clin Exp Res. 2019; doi: 10.1007/s40520-019-01413-1.

11. Kyle UG, Genton L, Hans D, Pichard C. Validation of a bioelectrical impedance analysis equation to predict appendicular skeletal muscle mass (ASMM). Clin Nutr. 2003;22(6):537-543; doi: 10.1016/S0261-5614(03) 00048-7.

12. Charlier R, Mertens E, Lefervre J, Thomis M. Muscle mass and muscle function over the adult life span: a cross-sectional study in Flemish adults. Arch Gerontol Geriatr. 2015;61(2):161-167; doi: 10.1016/j.archger.2015. 06.009 .

13. StatSoft Polska. Statistica 13.1. Available from: https:// www.statsoft.pl/statistica_13.

14. Fuller NJ, Elia M. Potential use of bioelectrical impedance of the 'whole body' and of body segments for the assessment of body composition: comparison with densitometry and anthropometry. Eur J Clin Nutr. 1989; 43(11):779-791.

15. Heymsfield SB, Smith R, Aulet M, Bensen B, Lichtman S, Wang J, et al. Appendicular skeletal muscle mass: measurement by dual-photon absorptiometry. Am J Clin Nutr. 1990;52(2):214-218; doi: 10.1093/ ajcn/52.2.214.

16. Webber CE, Barr RD. Age- and gender-dependent values of skeletal muscle mass in healthy children and adolescents. J Cachexia Sarcopenia Muscle. 2011;3(1):2529; doi: 10.1007/s13539-011-0042-6.

17. Gallagher D, Visser M, De Meersman RE, Sepúlveda D, Baumgartner RN, Pierson RN, et al. Appendicular skeletal muscle mass: effects of age, gender, and ethnicity. J Appl Physiol. 1997;83(1):229-239; doi: 10.1152/ jappl.1997.83.1.229. 
18. Carnevale V, Castriotta V, Piscitelli PA, Nieddu L, Mattera M, Guglielmi G, et al. Assessment of skeletal muscle mass in older people: comparison between 2 anthropometry-based methods and dual-energy X-ray absorptiometry. J Am Med Dir Assoc. 2018;19(9):793796; doi: 10.1016/j.jamda.2018.05.016.

19. Barbosa-Silva MCG, Barros AJD. Bioelectrical impedance analysis in clinical practice: a new perspective on its use beyond body composition equations. Curr Opin Clin Nutr Metab Care. 2005;8(3):311-317; doi: 10.1097/ 01.mco.0000165011.69943.39.

20. Slee A, Birch D, Stokoe D. Bioelectrical impedance vector analysis, phase-angle assessment and relationship with malnutrition risk in a cohort of frail older hospital patients in the United Kingdom. Nutrition. 2015;31(1): 132-137; doi: 10.1016/j.nut.2014.06.002.

21. Norman K, Stobäus N, Pirlich M, Bosy-Westphal A. Bioelectrical phase angle and impedance vector analysis - clinical relevance and applicability of impedance parameters. Clin Nutr. 2012;31(6):854-861; doi: 10.1016/j.clnu.2012.05.008.

22. Barbosa-Silva MCG, Barros AJD, Wang J, Heymsfield SB, Pierson RN Jr. Bioelectrical impedance analysis: population reference values for phase angle by age and sex. Am J Clin Nutr. 2005;82(1):49-52; doi: 10.1093/ajcn/82.1.49.

23. Bosy-Westphal A, Danielzik S, Dörhöfer RP, Later W, Wiese S, Müller MJ. Phase angle from bioelectrical impedance analysis: population reference values by age, sex, and body mass index. J Parenter Enteral Nutr. 2006;30(4):309-316; doi: 10.1177/014860710603000 4309.

24. Fukumoto Y, Ikezoe T, Yamada Y, Tsukagoshi R, Nakamura M, Mori N, et al. Skeletal muscle quality assessed from echo intensity is associated with muscle strength of middle-aged and elderly persons. Eur J Appl Physiol. 2012;112(4):1519-1525; doi: 10.1007/s00421-0112099-5.

25. Mckendry J, Breen L, Shad BJ, Greig CA. Muscle morphology and performance in master athletes: a systematic review and meta-analyses. Ageing Res Rev. 2018;45: 62-82; doi: 10.1016/j.arr.2018.04.007.

26. Garcia-Hermoso A, Tordecilla-Sanders A, Correa-Bautista JE, Peterson MD, Izquierdo M, Quino-Ávila AC, et al. Muscle strength cut-offs for the detection of metabolic syndrome in a nonrepresentative sample of collegiate students from Colombia. J Sport Health Sci. 2020;9(3):283-290; doi: 10.1016/j.jshs.2018.09.004.

27. Fraser BJ, Schmidt MD, Huynh QL, Dwyer T, Venn AJ, Magnussen CG. Tracking of muscular strength and power from youth to young adulthood: longitudinal findings from the Childhood Determinants of Adult Health Study. J Sci Med Sport. 2017;20(10):927-931; doi: 10.1016/j.jsams.2017.03.021.

28. Roberts HC, Denison HJ, Martin HJ, Patel HP, Syddall $\mathrm{H}$, Cooper $\mathrm{C}$, et al. A review of the measurement of grip strength in clinical and epidemiological studies: towards a standardised approach. Age Ageing. 2011; 40(4):423-429; doi: 10.1093/ageing/afr051.

29. Alizadehkhaiyat O, Hawkes DH, Kemp GJ, Howard A, Frostick SP. Muscle strength and its relationship with skeletal muscle mass indices as determined by segmental bio-impedance analysis. Eur J Appl Physiol. 2014; 114(1):177-185; doi: 10.1007/s00421-013-2764-y.

30. Silva AM, Shen W, Heo M, Gallagher D, Wang Z, Sardinha LB, et al. Ethnicity-related skeletal muscle differences across the lifespan. Am J Hum Biol. 2010;22(1): 76-82; doi: 10.1002/ajhb.20956. 